\title{
Mobile Based Notifiable Disease Surveillance - Case for Kenya
}

\author{
Christopher A Moturi \\ School of Computing and \\ Informatics \\ University of Nairobi \\ Nairobi, Kenya
}

\author{
Robert Muiruri Kinuthia \\ School of Computing and \\ Informatics \\ University of Nairobi \\ Nairobi, Kenya
}

\begin{abstract}
Identifying infectious disease outbreaks in their early stages is an important task, both for governments and international organizations working in the health sector. This study reviewed the notifiable disease reporting systems in Kenya with an aim of understanding their strengths and weaknesses. We evaluated the performance of the present notifiable disease reporting systems, assessed the preparedness of the disease notifiers for the task of reporting notifiable diseases, assessed the readiness of people who participate in disease reporting in the use of computers and mobile systems in disease surveillance, and we developed a mobile based disease surveillance prototype that can report and analyze notifiable disease data to recognize disease outbreaks early. The study targeted medical personnel who presently participate in the reporting and analysis of notifiable disease data in the Nairobi Metropolitan Region. Mobile systems are best to improve the disease reporting and analysis process because of their low cost, widespread use and minimum training required.
\end{abstract}

\section{Keywords}

Notifiable Disease, Notifiable Disease System, Disease Surveillance, Mobile Disease Surveillance.

\section{INTRODUCTION}

Disease outbreaks have the potential to give rise to a significant public health emergency because of their capacity to cause illness, death, fear and economic loss on a large scale. Public health officials are increasingly concerned about the spread of infectious diseases because of global travel, increased global trade in food and other commodities, and the emergence of antibiotic-resistant pathogens [1]. The lost productivity, missed educational opportunities and high health care costs caused by notifiable diseases directly impact families and communities. Massive increases in the volume and speed of international travel and trade that have occurred in recent years have increased opportunities for diseases to spread across international boundaries [2]. Worldwide disease reporting is critical if these diseases are to be effectively controlled and finally eradicated.

Each country will have its own arrangements for developing surveillance data according to its own needs and resources. WHO in partnership with various countries and support organizations maintains worldwide coverage of selected diseases and solicits the reporting. The Kenyan government list of infectious diseases that should be informed to medical authorities whenever they occur includes smallpox, plague, Asiatic cholera, yellow fever, sleeping sickness and any other disease which the Minister may, by order, declare to be a formidable epidemic disease as per the Public Health Act, 1961.

In 1998, the WHO Africa Region adopted a strategy called Integrated Disease Surveillance and Response [3]. In this strategy, countries aim at improving enabling capacities and support activities and select a number of priority diseases and health risk conditions from a list of the infectious diseases that affect African communities. Since then significant progress had been made in the development and use of national surveillance systems.

Identifying infectious disease outbreaks in their early stages makes the job of management and control of the disease easier and reduces their impact. A number of guidelines on the investigation and control of disease outbreaks have been documented [3, 4, 5]. Health providers at all levels need accurate and timely data in order to decide on the effectiveness of implemented policies and create new policies to note disease emergencies. The overriding public health goal is to bring the outbreak under control as quickly as possible, with as little social disruption as possible.

In the majority of cases, however, epidemics are generally well under way before authorities are notified and able to control the epidemic or mitigate its effects. In an effort to alleviate these epidemics in a world wide scale, WHO has identified a number of diseases that need to be notified to the authorities when they occur due to their ease of spreading named in today's world as notifiable diseases. Under the IHR [6] international law, countries work together to save lives under risk due to international spread of notifiable diseases and other health risks thereby strengthening the collective defences against the multiple and varied infectious health risks that today's globalized world is facing.

The Kenyan law recognizes the IHR [6] international law thereby providing for the reciprocal notification of outbreaks of any formidable epidemic or other disease of any other matter affecting the public health relations of Kenya with other countries.

Public health surveillance systems are essential for monitoring rates and distributions of infectious diseases so that outbreaks can be identified and controlled [7]. Rapidly identifying an infectious disease outbreak is critical, both for effective initiation of public health intervention measures and timely alerting of government agencies and the general public. According to IDSR [3] surveillance capacity for such detection can be costly and many countries lack the public health infrastructure to identify outbreaks at their earliest stages. 
Increasing infectious disease deaths, the emergence of new infections, and bioterrorism have made surveillance for infectious diseases a public health concern $[8,9]$. There is greater need of not only good laboratory and surveillance capacity, but also efficient ways of collecting and analyzing notifiable disease data. The internet and mobile communication systems have had impressive impact on the developments in health. Strong notifiable disease surveillance systems are essential for disease control [7]. The key benefits relate to the timeliness, cost effectiveness and broad-reaching of these technologies.

This paper evaluated the performance of the notifiable disease reporting system presently working in Kenya, assessed the readiness of people who participate in disease reporting in the use of computers and mobile systems in disease surveillance, and developed a mobile based disease surveillance prototype that can report and analyze notifiable disease data in order to recognize disease outbreaks early.

\section{RELATED WORK}

\subsection{Disease Surveillance}

The Technical Guidance for Integrated Disease Surveillance Response in the African Region prepared by WHO and CDC [3], disease surveillance is the ongoing systematic collection, analysis, and interpretation of health data. This includes the timely dissemination of the resulting information to those who need them for action. The main role of disease surveillance is to predict, observe and minimize the harm caused by outbreak, epidemic, and pandemic situations, as well as increase knowledge about which factors contribute to such circumstances. Disease surveillance is the foundation for prevention and control. The changes that have occurred in the health sector in the last twenty years have included the emergence of new diseases that have resulted in the need to review mechanisms for surveillance and response to these diseases. This is particularly so in the African Region, where it is recognized that while a lot of progress has been made towards improving national and regional capacity for effective surveillance and response, communicable diseases remain high priorities for national public health programs [3]. The communicable diseases that are remain high priorities for national public health programs include malaria, diarrhea, cholera, hemorrhagic fever, respiratory illness, HIV/AIDS, and tuberculosis. We sought to strengthen the availability of surveillance information to detect outbreaks in order take action to limit their impact on the health of those affected. Successful control of infectious diseases needs effective and efficient surveillance.

In order to monitor, evaluate and improve disease surveillance and response, IDSR [3] have recommended the following steps to be undertaken: identify targets and indicators, monitor the quality of surveillance activities at the district level, supervise surveillance and response activities, evaluate the surveillance and response system, and take action to improve surveillance and response system.

The World Health Organization Regional Office for Africa proposed an Integrated Disease Surveillance and Response approach for public health surveillance and response in the African Region in order to improve health facilities at district and national levels [3]. We sought to demonstrate how mobile phones can be used realize this and improve the flow of surveillance information between and within levels of the health system, increase involvement of clinicians in the surveillance system, and ultimately strengthen the capacity of Kenya to conduct effective surveillance activities.

\subsection{Use of Mobile Health in Disease Surveillance}

The past decade has seen growing interest in the field of mobile health (mHealth). MHealth is the provision of healthrelated services via mobile devices. There are a number of mHealth initiatives in public health currently being piloted and used in various parts of the world. We reviewed a number of these systems.

\subsubsection{National Notifiable Diseases Surveillance System (NNDSS)}

The National Notifiable Diseases Surveillance System (NNDSS) is a multifaceted Public Health disease surveillance system that provides gives powerful capabilities to public health officials to monitor the occurrence and spread of diseases (http://wwwn.cdc.gov/nndss/ ). The system provides functions for collection, management, analysis, interpretation and dissemination of health related data for nationally notifiable diseases; facilitates the development and maintenance of national standards; enable maintenance of the official national notifiable diseases statistics; provides detailed data to CDC programs to aid in identifying specific disease trends; and enhances the working with states and partners to implement and assess prevention and control programs.

\subsubsection{Pan-African mHealth initiative}

The GSMA Pan-African mHealth Programme seeks to engage mobile and health industries in order to unlock the potential of mobile technologies. This will deliver sustainable mHealth solutions that extend the reach and quality of healthcare while reducing costs.

http://www.gsma.com/connectedliving/mhealth/. The initiative aims to reduce fragmentation of mHealth services by creating a collaborative, integrated business framework to launch services. This will form the business framework for scaled and sustainable solutions to be replicated across multiple countries, and align health and mobile industries around common goals and reduce barriers preventing the sustainable and scaled integration of mobile into health.

(http://www.gsma.com/mobilefordevelopment/programmes/m health/pan-african-mhealth-initiative ).

\subsubsection{Global mHealth Initiative}

The John Hopkins University Global mHealth Initiative has 125 registered projects spanning across the entire institution (http://www.jhumhealth.org/ ). They include: Use of SMS reminders to improve timely vaccine coverage in rural Africa; Integrated mobile phone based data system that links rural community health workers and their clients (mCare); Mobile application package that can rapidly capture, analyze and record water quality parameters in remote regions; Raising awareness and marketing male medical circumcision to reduce HIV transmission risk in sub-Saharan Africa (Brothers for Life); Communication program designed to investigate the low bandwidth potential of SMS technology to facilitate communication, enable situational awareness, and rescue survivors during disasters (Disaster Link); A virtual, interactive, mobile-phone based "Immunization Record" to Improve vaccination rates in rural Bangladesh (mTikka); Develop virtual patient records with regards to localized language and semantics (Mobile EMR); A mobile intervention tool designed to reduce hospital readmissions; SMS service to provides information on male circumcision; Information and counseling services via telephone for people 
who want to know more about HIV/AIDS and other STD's (Info SIDA); Empowering pregnant women and their partners to take the steps necessary for a healthy pregnancy and safe delivery through a national, multi-channeled strategic behavior change communication campaign (Wazazi Nipende); Empowering women to initiate use of modern methods of family planning and encourage male support of family planning (Jiamini); and communications between hospitals and isolate villages in Malawi (K4Health Malawi).

\subsubsection{National Electronic Disease Surveillance System (NEDSS)}

The National Electronic Disease Surveillance System is a standards-based approach to connect public health and clinical medicine. The initiative focuses on the use of data and information systems standards to advance the development of most suitable surveillance systems at the at national and local levels. (http://www.ncbi.nlm.nih.gov/pubmed/11713753 ).

\subsubsection{SIM-pill}

The SIM-pill system monitors patient medication schedule and intake of medication and reminds patients and care givers by sending a text message (http://www.simpill.com /). In the pilot stage in South Africa, $90 \%$ of patients complied with their TB medication compared to $22 \%$ to $60 \%$ take-up without it.

\subsubsection{Text to Change}

The Text to Change is an example of how a simple application can make a big difference. This service communicates interactive and incentive-based SMS projects, targeting for example HIV/AIDS, malaria and reproductive health issues (http://www.texttochange.org)

\subsubsection{Jamii Smart Kenya}

The Jamii Smart Kenya is a World Vision Kenya mHealth initiative being implemented in partnership with Safaricom, Amref, Care, and World Vision while Aga Khan University is implementing research and evaluation of the initiative to provide strategic guidance for its implementation (http://www.wvi.org/health/mhealth-kenya ). The mobile solution uses web portals and SMS to deliver the clinical components for mother and under 5 case management and link it to a community health monitoring and evaluation system.

\subsubsection{Kenya Integrated Mobile $\mathrm{MNCH}$ Information Platform}

The Kenya Integrated Mobile MNCH Information Platform (KimMNCHip) is a national-scale mHealth initiative to offer pregnant women in Kenya more choice, control and care during their pregnancy, and improved medical care for them and their babies during and after delivery (http://healthmarketinnovations.org/program/kenyaintegrated-mobile-mnch-information-platform-kimmnchip ).

\subsubsection{National Electronic System for Disease Surveillance in Rwanda (eIDSR)}

Rwanda has deployed a national Electronic System for Disease Surveillance (eIDSR) that has enhanced its preparedness to identify and respond to outbreaks and prevent epidemics [10]. With funding from US Centers for Disease
Control, the mobile technology based system was developed and implemented by Voxiva in 2013.

\subsubsection{Other Related Work}

Burdakov et al. [11] demonstrated how one-health surveillance in medical, veterinary and environmental sectors can be improved with Electronic Integrated Disease Surveillance System using an example from Kazakhstan.

It has been shown how Smartphone applications have the potential to generate real-time disease surveillance data that may augment current methods [12]. They developed novel and free Smartphone application, ClickClinica, to try to increase the identification of major infectious diseases and other acute medical presentations and improve our understanding of clinical practice.

Asif et al. [13] have developed a web-GIS based system for public health surveillance that efficiently provides the maps and charts in real time through internet. This decision support system for public health surveillance was tested with data from Thailand proved useful to decision makers and public health officials for analysis and taking preventive actions.

Koopmans [14] has discussed innovations in diagnostics and surveillance aimed at early detection of unusual disease in view of new pathogens that continue to emerge and the increased connectedness of populations across the globe through international travel and trade.

A comparative study of the existing notifiable disease surveillance systems of Sri Lanka and the UK has been done by [15]. They explored the features that would be applied to develop an information system suitable for the surveillance of notifiable diseases in Sri Lanka. They recommend receiving notifications from various sources, involvement of laboratories in disease surveillance activities, and computerizing the existing surveillance system for notification to enhance the completeness and timeliness of reporting.

In [16] there is an assessment of the emergence, development, and value of regional infectious disease surveillance networks that neighboring countries worldwide are organizing to control cross-border outbreaks at their source. The paper is an instructive framework for other countries who decide to launch such networks considering that new technologies and emerging threats bring countries even closer together.

There are several international scientific databases available with integrated disease surveillance systems. They include the

Global Science Gateway WorldWideScience.org and Science.gov:

(http://worldwidescience.org/topicpages/e/electronic+disease+ surveillance.html)

(http://www.science.gov/topicpages/i/infectious+disease+surv eillance.html).

\subsection{Use of Mobile Health in Kenya}

Among these initiatives, SMS stands out as being the most advantageous in terms of its cost-effectiveness, scalability, convenience, broad reach, and widespread popularity in the developing world. Nyatanyi et al. [10] for example, have described how this has been rolled out nationally in Rwanda. SMS alerts have proven particularly effective in targeting hard-to-reach populations and rural areas where the absence of clinics, lack of healthcare workers, and limited access to health-related information all too often prevent people from making informed decisions about their health. 
These initiatives are closing the information gap that currently exists for patient data in the developing world, enabling public officials to gauge the effectiveness of healthcare programs, allocate resources more efficiently, and adjust policies accordingly. This is more empowered by the fact that the price of short message service has dropped by more than $50 \%$ in Kenya, and most other African countries, in the last few years. Mobile phone network GSM, GPRS data connectivity provides a viable national wide and cost effective mode of connecting through the internet to any server and this is an option that needs to be actively explored [17].

In Kenya, a country with a population of around 42 million, there is $51 \%$ coverage of mobile communication. The growth in this sector is expected to grow at a higher momentum due to the lower cost of acquiring handsets and dropping of calling SMS/costs [18].

A national cross-sectional survey in public health facilities in Kenya was undertaken by [19]. Mobile phone ownership and SMS use is widespread among health workers in the public sector. Disparities exist with respect to gender, age, education, literacy, urbanization and poverty. Some of the disparities on SMS use can be addressed through the modalities of mHealth interventions and enhanced implementation processes while further growth in mobile phone penetration is needed to reduce the ownership gap.

In Kenya, the delay before the national office receives any information on the notifiable disease data from the districts/counties averages 10 days. Recent evidence from research in developing countries shows that data collection process can be more effective, efficient and reliable if conducted through mobile phones rather than the present paper format. There is growing evidence showing the potential of mobile systems to radically improve healthcare services, even in some of the most remote environments.

\section{METHODOLOGY}

\subsection{Research Design}

The study was conducted in the Nairobi Metropolitan comprising of Nairobi, Kiambu, Machakos and Kajiado counties. This is a region of the country with an approximate population of 5,640,273, covering 32,000 square $\mathrm{km}$ and contributes over $50 \%$ to the GDP of Kenya. In addition to hospitals, mortuaries were included because of the high number of people who do not attend hospitals when they are sick and in some cases death may have been caused by an infectious disease. The hospitals are run by ministry of health, faith based organizations, local authorities, NGOs, and private institutions. In each of the hospitals, staff involved directly in the testing and reporting of notifiable diseases were targeted. These included doctors, clinical officers, laboratory technicians, surveillance officers, post mortem officers, and pathologists.

\subsection{Data Collection and Analysis}

Data was obtained from participants using semi-structured questionnaires that had open and close-ended questions. Some of the questionnaires were filled in the presence of the researchers thereby reducing the ambiguity that goes with questionnaires. Forms used by the Ministry of Health to fill and pass notifiable disease data to the various levels were reviewed. To test their reliability, the questionnaire was pretested in 10 hospitals, two each representing the various categories. After scrutiny to identify ambiguity, misleading questions or instructions and suggested improvements, various changes were made to questionnaires.
The key questions in the questionnaire were: computers and related media available at the hospitals, the usage of mobile phones, information required for disease surveillance, perceptions and attitudes on the reporting of notifiable disease outbreaks in the hospitals, benefits and challenges of infectious disease reporting system, and suggestions to improve the surveillance system of infectious diseases.

A stratified random sampling procedure was used to determine the number of hospitals, keeping in view the two causes of incorrect inferences i.e. systematic bias and sampling error [20]. To ensure that the sample was representative of a population about which there was a fair amount of information available, the population was divided into sub-groups representing government hospitals, private hospitals (privately owned and faith sponsored), doctors, clinical officers, lab technicians, surveillance officers, post mortem officers, and pathologists. The sample size was determined by the relative size of the hospital.

A pre-coding procedure was adopted for closed questions with predetermined answers to each question. Various approaches were used for coding open-ended questions which produced multiple responses. This depended on the question and the number of replies given. The data gathered from the structured questionnaires was edited for completeness and consistency coding to enable the responses to be grouped into categories. Microsoft Excel was used to analyze the data.

\section{RESULTS AND DISCUSSION 4.1 Results}

Out of the 702 hospitals in the study, 99 questionnaires were sent out and 88 returned, distributed in the Nairobi Metropolitan in terms of districts as follows: Kiambu East (6), Kiambu West (10), Machakos (12), Nairobi East (14), Nairobi North (24), Nairobi West (14) and Kajiado (8). In terms of ownership Private Hospital account for (52\%), Faith Based (19\%), Ministry (8\%), Local Authorities (17\%) and NGOs account for $(4 \%)$. There were $63 \%$ male and $37 \%$ female. The levels of educations were as follows: $\mathrm{PhD}(2 \%)$, Masters (15\%), Bachelors (41\%), Diploma (40\%), and Certificate $(2 \%)$. Professionally there were Doctors $(35 \%)$, Clinical Officer (31\%), Lab Technician (28\%), Surveillance Officer (3\%), and others such Microbiologists and Pathologists (3\%).

\subsubsection{Notification Disease Reporting Systems}

Assessment of the notification disease reporting systems show that the most common means of learning about disease outbreaks are from the respective hospitals, word of mouth and TVs (Fig. 1). 34\% of the respondents rated themselves as either 'Good' or 'Very Good', 39\% rated themselves as 'Average' in disease reporting while 27\% rated themselves negatively in disease reporting.

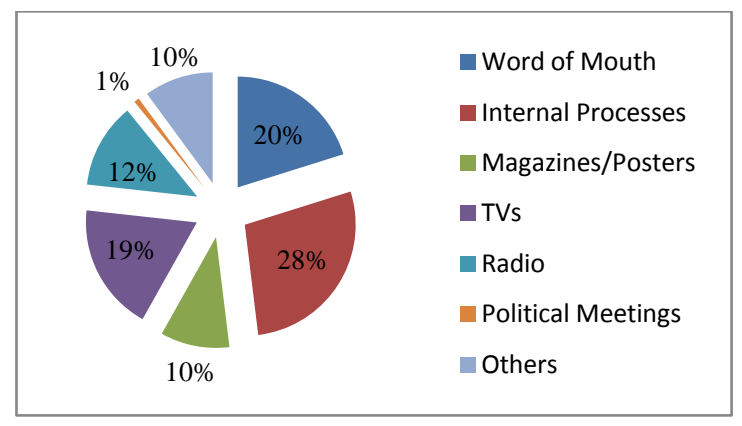

Fig. 1: Notification Disease Reporting Systems 
About $45 \%$ of the respondents considered the paper based reporting system time consuming while $34 \%$ considered lack of feedback as the biggest challenge in reporting notifiable diseases. 57\% considered feedback as the best way to encourage disease reporting while $35 \%$ considered payment of bonuses as the best method. $2 \%$ thought that compulsory reporting could improve reporting while another group adding up to $6 \%$ had various other ways of encouraging reporting e.g. having specific people employed for notifiable disease reporting in each hospital.

About half of the respondents proposed that the best way to improve disease reporting was to introduce technology to the health sector while a quarter proposed feedback to the person/hospital sending. A smaller proportion chose payment of bonuses as the best way to improve the reporting of notifiable diseases (Fig. 2).

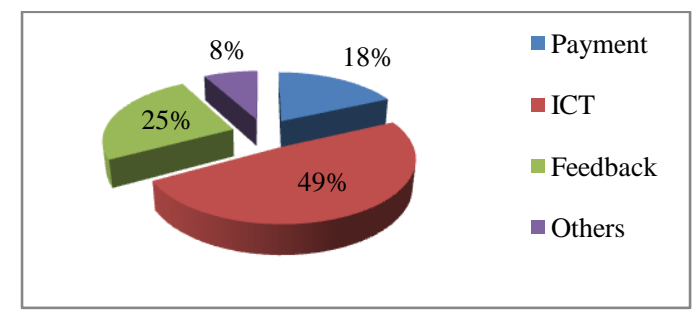

Fig.2: Improve Reporting of Notifiable Diseases

\subsubsection{Preparedness of the Disease Notifiers}

Only $44 \%$ of the respondents had undergone formal training on how to report notifiable diseases while $56 \%$ had not. Out of those who had received training, the source of funds for the training was Ministry of Health (63\%) and the hospital (32\%). None were self sponsored.

\subsubsection{Readiness in use of ICT}

The distribution of available ICT facilities was as follows: $53 \%$ of the stations had at least a computer, while $51 \%$ of the stations with computers had network facilities and $38 \%$ of the stations with computers had internet connection. None of the stations had PDAs. Nearly all the respondents (98\%) noted that the easiest technology to access in their work environment is the mobile phone as shown in Fig 3, Fig 4 and Fig 5. This is out of computers, network, internet and mobile phone. $98 \%$ of the respondents had functional mobile phones at the time, with average ownership duration of 5 years. $52 \%$ of the respondents had ICT training while $48 \%$ had no formal ICT training; with the majority $(65 \%)$ having sponsored themselves while $35 \%$ had been sponsored by the organization or former organization they were working for.

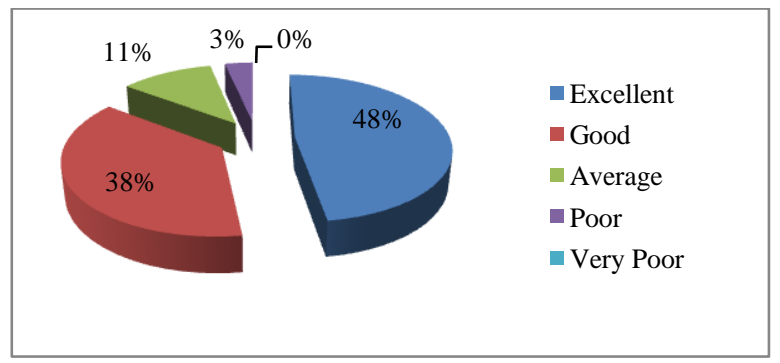

Fig 3: Ability to Make Calls

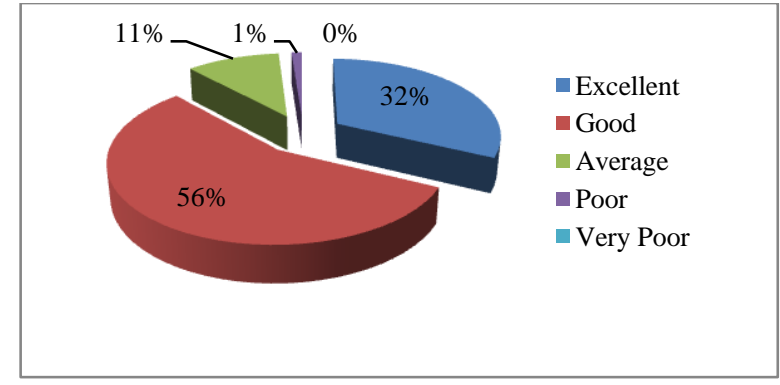

Fig. 4: Ability to Use SMS

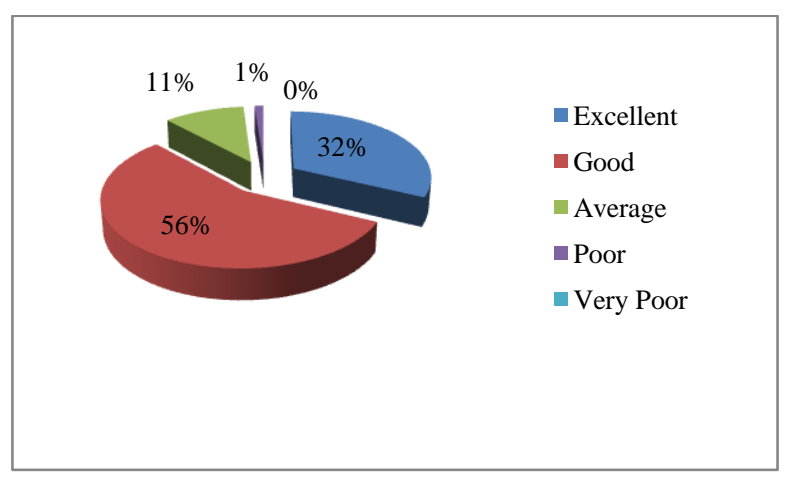

Fig 5: Ability to Send and Receive Money

\subsection{Discussion}

The study established that the present disease reporting system which is paper based is very inefficient as takes up to ten days before the national office knows of a notifiable disease occurrence in the hospitals. This duration is considered extremely long noting that some diseases can kill in less than 48 hour. There is no means of feedback to the hospitals once a disease is reported. To close this gap of lack of feedback most hospitals covered have created local disease surveillance units where recognition of most disease outbreaks is done.

It was established that the health workers are adequately trained on the job of reporting diseases with more than two thirds of the respondents having undergone formal training on how to report notifiable diseases. The research recognized the collaborative effort being put in by NGO's and the government in training. To improve this most hospitals have their own local training to recognize notifiable diseases. This is mostly done by those who attend national training programs with an aim of empowering their colleagues. The work of recognizing disease outbreaks has been delegated to surveillance departments and information officers in the hospitals.

The study established that the existing computers and information resources in hospitals are not adequate to fully support reporting of notifiable diseases as close to half of the hospitals did not have the basic requirement (a computer and internet connection). Government sponsored hospitals seem to be the most underfunded in ICT. There is willingness to improve the reporting of notifiable diseases by use of technology, but the government does not seem to be giving this option much emphasis as more than two thirds of the people who have formal training in ICT sponsored themselves.

Nearly all the medical staff had mobile phones and mobile phones are more accessible in the work environment than a computer. About half of the respondents noted that 
introduction of technology would improve the disease reporting in the country. It is worth noting that the efficiency in using mobile phones of various health personnel is high and a proposal of using mobile systems is highly encouraged, noting the limited amount of training that may be required and major costs of structuring and setting up have been borne by the mobile companies. It is also recommended that, as we try to send data to the national level, the district level needs to be empowered because response in terms of verification and appropriate action are fastest from the district level.

\subsection{Mobile based Disease Surveillance Prototype System}

We developed a prototype system in the form of an early warning disease response system. The prototype was developing taking into consideration limitation of resources in developing countries, such as Kenya, as suggested by the generic architecture proposed by [21]. The prototype provides medical notifiers in the field with an interface to report notifiable diseases. The user must first registers their SIM card by sending a message to the servers. The user can then enter data about a disease case which has been recognized in the field in a given format. When the message has been saved, a confirmation message is sent to the sender. A copy of the message is then sent to the district office. This enables the district office to respond faster as they are closer to the source of the disease. On sending a message to the national office server, a confirmation message is also sent. The system carries out analysis of data so that disease outbreaks can be recognized. This includes comparing disease data of different times in order to recognize an outbreak. Various reports including charts can be generated.

The non-functional requirements include easy to use interface to enable the user to carry out basic tasks, reliability, integrity, security to allow only authorized users, and good usability such as similar screens. The conceptual design and the system flowchart of the systems are shown in Fig 6 and Fig 7 below.

Among the inbuilt security features is a mobile number and registration number based authentication. The system checks if the mobile number is registered and if so, then it will check the associated person id, other details about where they work e.g. work place, occupation and permission to send SMS. The person registered cannot use any other mobile phone number.

In the development of the prototype, various types of testing were carried. Module testing focused on verification effort on the smallest unit of software design. Using the detail design description as a guide, important control paths were tested to uncover errors within the boundary of the module. Regression testing involved testing and error correction process such that the system does not create more errors out of the error correction process. The integration testing involved a systematic technique for constructing the program structure while at the same time conducting tests to uncover errors associated with interfacing.

The system requires a minimum of Windows server 2003, 2008. Other operating systems e.g. windows XP and Windows 7 will also work well. It requires support software such as internet explorer or Mozilla, acrobat and Microsoft Office Suite 2003/2007.

The analysis of the early warning disease response systems shows that the public health sector would benefit. This is particularly so when you consider that at present, data is sent through manual forms.

An assessment of the technology to be applied showed that the technology is mature and available. The technology is also supported by the number of mobile devices in Kenya with the presence of four mobile based companies.

The working of the software is within the legal framework in the Kenyan health sector. Issues of confidentiality and privacy of personal health data have been taken care of in the design aspects. Cost analysis showed that it is more cost effective to implement. The benefits greatly outweigh the costs incurred in the design and implementation. The study showed that the system could be developed and implemented within an acceptable period of time. 


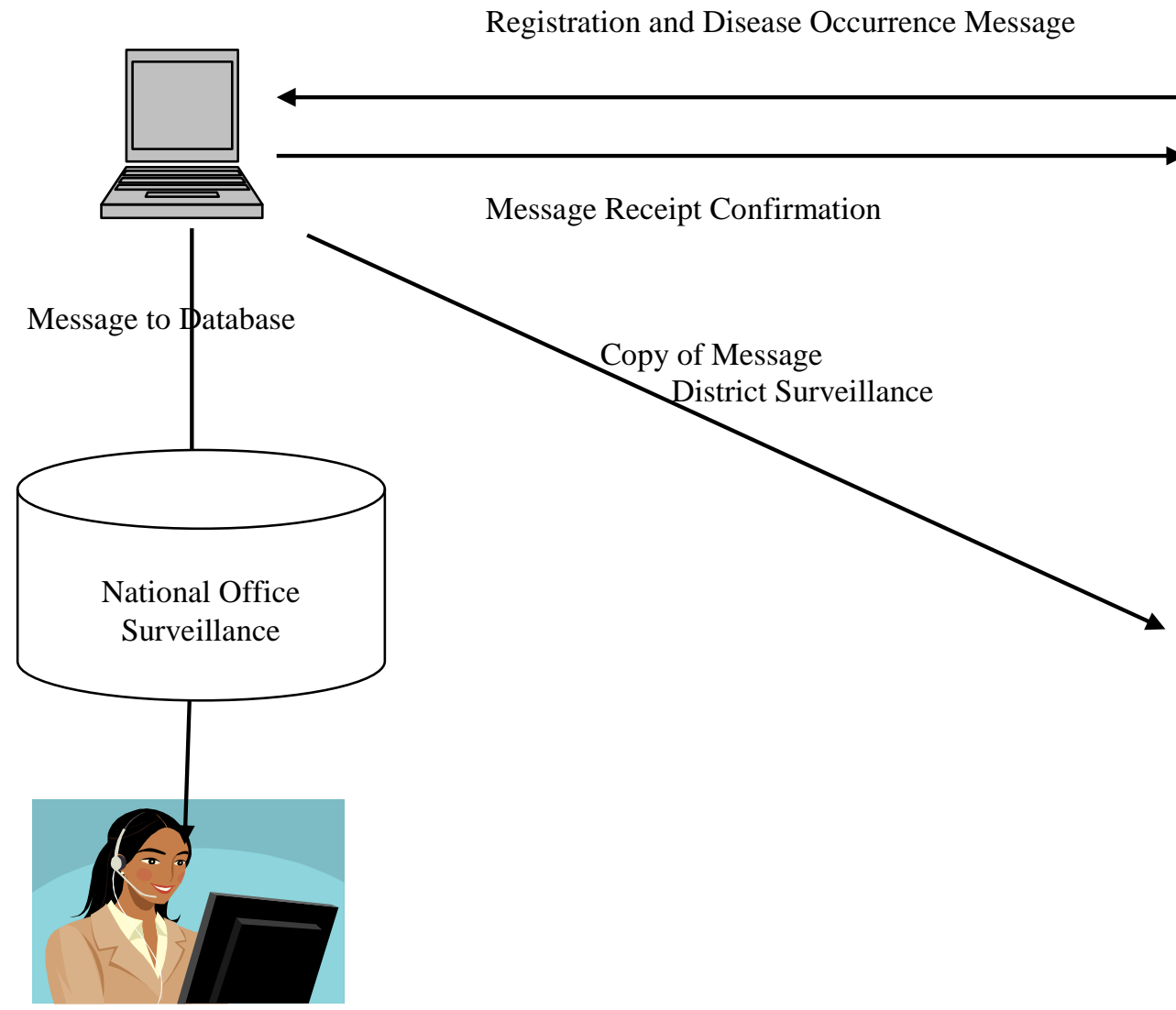

Field Disease Notification

Fig 6: Conceptual Design of the Mobile Based Disease Surveillance Prototype System

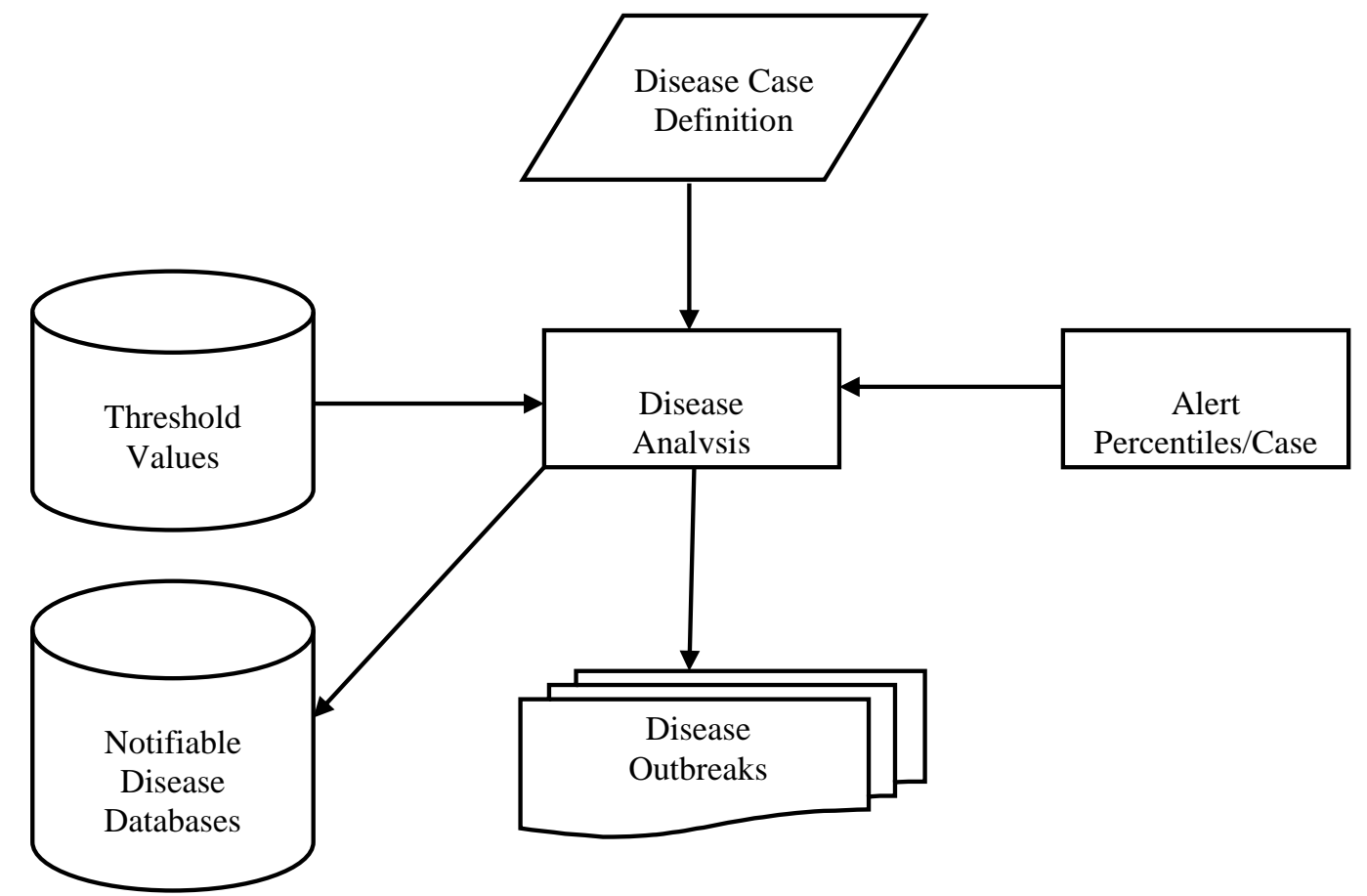

Fig 7: System Flowchart of the Mobile Based Disease Surveillance Prototype System

\section{CONCLUSION}

There is need for improvements and enhancements in the dissemination on national notifiable diseases surveillance systems all over the world [22, 23]. Lessons from other African countries such as Rwanda [10] show how a developing country can deploy a national integrated disease surveillance and response system. This paper assessed notifiable disease reporting systems in Kenya, the preparedness of the disease notifiers, and readiness of hospitals and people who participate in disease reporting in the use of computers and mobile systems in disease surveillance. The mobile based notifiable disease reporting prototype developed demonstrates practically the reporting 
and analysis of notifiable diseases for reporting. This would enable decisions about disease outbreaks to made thereby appropriate action taken to alleviate epidemics.

Future extension will be the creation of surveillance software for infectious diseases that are common to both human and animals. Such a system has been implemented in Sri Lanka [24]. This is supported by the fact that most emerging disease such as H1N1, AIDs and SARS, which are major threats in the world today, have their origin in animals. It is also noted that the IDSR strategy that takes into account the One WorldOne Health perspective which is a strategy that addresses events at the intersection of human, domestic animal, wildlife, and ecosystem health [3].

\section{REFERENCES}

[1] Lister, S.A. (2005). An Overview of the U.S. Public Health System in the Context of Emergency Preparedness, Congressional Research Service. The Library of Congress. Available at https://www.fas.org/sgp/crs/homesec/RL31719.pdf

[2] GAO (2001). Global Health: Challenges in Improving Infectious Disease Surveillance Systems. United States General Accounting Office. Available at http://www.gao.gov/new.items/d01722.pdf

[3] IDSR (2010). Technical Guidance for Integrated Disease Surveillance Response in the African Region. WHO \& CDC, 2nd edition. Available at

[4] http://www.cdc.gov/globalhealth/dphswd/idsr/pdf/Techni cal\%20Guidelines/IDSR\%20Technical\%20Guidelines\% 202nd\%20Edition_2010_English

[5] WHO (2005). Outbreak Communication Guidelines. Available at http://www.who.int/csr/resources/publications/WHO_CD S_2005_28en.pdf?ua $=1$

[6] ESR (2012). Guidelines for the Investigation and Control of Disease Outbreaks. Institute of Environmental Science \& Research Limited. Available at https://surv.esr.cri.nz/episurv/Manuals/GuidelinesForInv estigatingCommDiseaseOBs.pdf

[7] IHR (2005). International Health Regulations. WHO, Second Edition. Available at http://whqlibdoc.who.int/publications/2008/9789241580 410_eng.pdf

[8] Trepka, M.J., Zhang, G., Leguen, F., (2008). An Intervention to Improve Notifiable Disease Reporting Using Ambulatory Clinics. Cambridge University Press. Available http://www.ncbi.nlm.nih.gov/pubmed/18466662

[9] Wolfe, M.I, Nolte, K.B. and Yoon, S.S. (2004). Fatal Infectious Disease Surveillance in a Medical Examiner Database, Emerging Infectious Diseases. CDC, Vol 10, No. $1 . \quad$ Available at http://wwwnc.cdc.gov/eid/article/10/1/020764_article.htm

[10] Kimball, A.M., MD, French, H.M., Ungchusak, K. and Taylor, T. (2008). Regional Infectious Disease Surveillance Networks and their Potential to Facilitate the Implementation of the International Health Regulations. Regional Surveillance Networks, pp 14601470.

Available
http://download.thelancet.com/flatcontentassets/H1N1flu/surveillance/surveillance-2.pdf

[11] Nyatanyi, T., Kabeja, A. Asiimwe, A., Binagwaho, A. Koama, J. B., Johnson, P. and Kayumba, K. (2013). A National Electronic System for Disease Surveillance in Rwanda (eIDSR): Lessons Learned from a Successful Implementation. Proceedings of the International Society for Disease Surveillance (ISDS) Conference. Available at http://www.syndromic.org/component/content/article/25/ 373

[12] Burdakov, A.V. Ukharov, A.O. and Wahl, T.G. (2013). One Health Surveillance with Electronic Integrated Disease Surveillance System. Online Journal of Public Health Informatics, 5(1), pp 199. Available at http://www.ncbi.nlm.nih.gov/pmc/articles/PMC3692852/ pdf/ojphi-05-199.pdf

[13] Michael, B.D.M. Geleta, D. (2013). Development of ClickClinica: a novel smartphone application to generate real-time global disease surveillance and clinical practice data. BMC Medical Informatics and Decision Making, 13(70). Available http://www.biomedcentral.com/1472-6947/13/70

[14] Asif, M., Tripathi, N.K, and Ahmed, S. (2013). Towards Near Real Time Public Health Surveillance (A Decision Support System for Public Health Surveillance). International Journal of Computer Applications, Vol 61, No. 21, pp 45-50. Available at http://research.ijcaonline.org/volume61/number21/pxc38 85083.pdf

[15] Koopmans, M. (2013). Surveillance strategy for early detection of unusual infectious disease events. Current Opinion in Virology, Vol 2, Issue 2, pp 185-191.

[16] Chandrasekar, K., Mahesan, S., Bath, P.A., (2013). Notifiable disease surveillance in Sri Lanka and the United Kingdom: a comparative study. Sri Lanka Journal of Bio-Medical Informatics, 4(1):14-22.

[17] Bond, K.C., Macfarlane S.B., Burke, C., Ungchusak,K., and Wibulpolprasert, S., (2013). The Evolution and Expansion of Regional Disease Surveillance Networks and Their Role in Mitigating the Threat of Infectious Disease Outbreaks. Emerging Health Threats Journal, pp 1-10. Available at http://www.ncbi.nlm.nih.gov/pubmed/23362414

[18] MOH (2009). Review of Software in the Health Sector. Technical Report, Ministry of Health, Kenya.

[19] CCK (2010). The Kenya Information and Communications (Universal Access and Service) Regulations.

[20] Zurovac, D., Otieno, G., Kigen S., Mbithi, A., Muturi, A., Snow, R.W. and Nyandigisi, A. (2013). Ownership and use of mobile phones among health workers, caregivers of sick children and adult patients in Kenya: cross-sectional national survey. Globalization and Health, 9 (20), pp 1-7. Available at http://www.ncbi.nlm.nih.gov/pubmed/2367230

[21] Kothari, C. R: (2004). Research Methodologies, Methods and Techniques 2nd Edition. New Age International publishers.

[22] Fondze, N.R., Texier, G., Tchendjou, P., Kouamou, G.E., Njouom, R., Demanou, M., Tchuente, M., (2013). An 
Early Warning Surveillance Platform for Developing Countries. Proceedings of the International Society for Disease Surveillance (ISDS) Conference. Available at http://www.syndromic.org/storage/documents/isdsconference/2013-

Conference/abstracts/an\%20early\%20warning\%20survei llance $\% 20$ platform $\% 20$ for $\% 20$ developing $\% 20$ countries. pdf

[23] Grigoryan, A and Jajosky, R.A., (2013). Environmental Scan of the US States' and Other Countries' Notifiable Disease Provisional Data Online Display: Results and Recommendations. International Society for Disease Surveillance (ISDS) Conference. Available at http://www.syndromic.org/storage/documents/isdsconference/2013-

Conference/abstracts/environmental $\% 20$ scan $\% 20$ of $\% 20 \mathrm{t}$ he\%20us\%20states\%20and\%20other\%20countries.pdf

[24] Clifford, D.G., (2007). The Voxiva Story. Available at http://www.mit.edu/ gari/teaching/prahalad_sec_Va.pdf

[25] Robertson, C. Sawford, K., Daniel, S.L.A., Nelson, T.A. and Stephen, C. (2010). Mobile Phone-based Infectious Disease Surveillance System, Sri Lanka. Emerging Infectious Diseases. 16(10), pp 1524-1531. Available at http://wwwnc.cdc.gov/eid/article/16/10/pdfs/10-0249.pdf

[26] National Electronic Disease Surveillance System Working Group (2001). National Electronic Disease
Surveillance System (NEDSS): a standards-based approach to connect public health and clinical medicine. J Public Health Manag Pract. 7(6): pp 43-50. Available at http://www.ncbi.nlm.nih.gov/pubmed/11713753

[27] http://wwwn.cdc.gov/nndss/ Accessed March 17, 2013

[28] http://www.jhumhealth.org/. Accessed August 17, 2013

[29] http://www.simpill.com. Accessed August 18, 2013

[30] http://www.gsma.com/connectedliving/mhealth/ Accessed January 15, 2014

[31] http://www.gsma.com/mobilefordevelopment/programm es/mhealth/pan-african-mhealth-initiative Accessed August 17, 2013

[32] http://www.texttochange.org Accessed August 18, 2013

[33] (http://healthmarketinnovations.org/program/kenyaintegrated-mobile-mnch-information-platformkimmnchip Accessed January 17, 2014

[34] http://worldwidescience.org/topicpages/e/electronic+dise ase+surveillance.html Accessed March 1, 2014

[35] http://www.science.gov/topicpages/i/infectious+disease+ surveillance.html Accessed March 1, 2014

[36] http://www.wvi.org/health/mhealth-kenya Accessed February 12, 2014 